Copyright (C 2014 IEEE. Personal use of this material is permitted. Permission from IEEE must be obtained for all other uses, in any current or future media, including reprinting/republishing this material for advertising or promotional purposes, creating new collective works, for resale or redistribution to servers or lists, or reuse of any copyrighted component of this work in other works. 


\title{
A Novel Instructional Approach to the Design of Standard Controllers: Using Inversion Formulae
}

\author{
Lorenzo Ntogramatzidis, Roberto Zanasi, Member, IEEE, and Stefania Cuoghi,
}

\begin{abstract}
This paper describes a range of design techniques for standard compensators (Lead-Lag networks and PID controllers) that have been applied to the teaching of many undergraduate control courses throughout Italy over the last twenty years, but that have received little attention elsewhere. These techniques hinge upon a set of simple formulae - herein referred to as Inversion Formulae - for the computation of the parameters of the controller as functions of the typical specifications of steady-state performance, stability margins and crossover frequency.
\end{abstract}

Index Terms-crossover frequencies, Lead and Lag networks, PID controllers, stability margins, steady-state performance.

\section{INTRODUCTION}

Automatic control is a key course in the curricula of electrical, electronic and communication engineers. Its syllabus is usually divided into two parts: the first focuses on the concepts of transfer function, frequency response, Bode/Nyquist/Nichols diagrams and root locus; the second deals with compensator design. The standard compensators presented in every control course and textbook belong to two families: Lead/Lag (or phase correction) networks and PID controllers. The structures of these controllers are simple, which partly justifies the practice of employing them as prototype examples to illustrate the various synthesis methods in such courses. The importance of these compensators resides also in their relevance in applications. It is often argued that $90 \%$ of the compensators used in industry are PID controllers alone [1].

The common trend in both traditional and modern approaches to control education is to formulate the feedback control problem as one in which the design specifications are first expressed using time domain parameters of the response (overshoot, undershoot, steady-state accuracy, etc). These requirements are then transformed into frequency domain specifications (DC gain, bandwidth, resonant peak, phase and gain margins, crossover frequencies, etc). The tuning techniques introduced in the majority of control

Ntogramatzidis is with the Department of Mathematics and Statistics, Curtin University, Perth, WA, Australia (L.Ntogramatzidis@ curtin.edu.au).

Drs Zanasi and Cuoghi are with the DII-Information Engineering Department, University of Modena and Reggio Emilia, Modena, Italy (roberto.zanasi@unimore.it, stefania.cuoghi@unimore.it).

This work was partially supported by the Australian Research Council under the grant FT120100604.

Manuscript received April 19, 2005; revised December 27, 2012. courses are based on trial-and-error methods usually applied on the Bode, Nyquist or Nichols plots. When applying these methods within the context of written exercises (assignments, tests, final exams), there are essentially two sources of approximation that need to be taken into account. First, the method itself is approximate. Second, within the context of a written exercise, students can only draw asymptotic Bode plots, which are themselves an approximation of the real plots.

In this paper, a numerical and graphical methodology is presented which has been successfully employed in both an educational and practical context for controller design from standard specifications such as steady-state performance, gain and phase crossover frequencies, phase and gain margins. This method is based on the so-called Inversion Formulae, which deliver the parameters of the compensator as an explicit function of the specifications. These formulae first appeared for generic first-order compensators in [2], and their geometric interpretation in the context of control feedback design was explained in [3]. The versatility of the approach in [2] - adapted to the discrete case in [4] - also enables this technique to be applied to second and third-order compensators as well as to scenarios with different specifications. Surprisingly, the pioneering paper [2] has never been cited in the literature, nor is there evidence - to the best of the authors' knowledge - that this method has been extensively employed in an educational context, which may explain why this method is still relatively unknown to the wider scientific community. A significant exception is the Italian control literature; this technique appears in the Italian control textbook [5], which over the past twenty years has been the most popular by far of the control textbooks used in courses in Italian tertiary education. The same technique later appeared in other Italian university-level textbooks, e.g., [6], but the success of this technique for educational purposes has so far remained mainly confined within the Italian control community.

The aim of this paper is to present this technique within a new learning context, to make its potential in control systems design education clear to a wider scientific audience. It is also shown that a vast array of written exercises can be devised using this approach, that cannot be tackled by resorting to the standard techniques; this is demonstrated with a running example involving a number of questions that 
can be addressed and solved in closed form. The usefulness of this didactic approach lies in two areas. First, the entire synthesis procedure of the first and the vast majority of second order controllers encountered in control education can be carried out with pen, paper and a scientific calculator; it is therefore very suitable for use in all forms of written questions and exercises. Second, the graphical interpretation of the design procedure based on the Inversion Formulae on Nyquist, Bode or Nichols plots creates important cognitive links to other topics encountered in a control course, [7]. Indeed, enhancing the connection between several underlying principles of feedback control theory has been recognised to lead to a more persistent knowledge, as shown in the socalled constructive learning approach, [8], [9].

Unlike the case of traditional design methodologies, the feasibility of the design procedure based on the Inversion Formulae can be checked a priori. Moreover, this method can be implemented as an extremely simple and insightful algorithm, for example by using MATLAB $^{\circledR}$. In this way students can be actively involved in the creation of a tool for the synthesis of regulators, on the basis of the example presented in this paper, which plays an active role in their learning process. It is also shown that the design method based on the Inversion Formulae has a fundamental graphical counterpart; this adds a further dimension to the learning experience of the synthesis of standard compensators, as highlighted in [7].

\section{FORMULATION OF THE CONTROL PROBLEM}

Consider the first feedback scheme in Fig. 1, where $G(s)$ and $C(s)$ are the transfer functions of the plant and of the controller, respectively, and where $r, y, e$ and $u$ are the reference signal, the output, the tracking error and the control input, respectively. All the poles of $G(s)$ are assumed to be in the left half plane except for, possibly, a pole at the origin.

Problem 2.1: Find a controller $C(s)$ such that the steady-state requirements on $e(t)$ are satisfied, and the gain crossover frequency and phase margin of the open-loop transfer function $L(s) \stackrel{\text { def }}{=} C(s) G(s)$ are $\omega_{g}$ and $\mathrm{PM}$, respectively.

Problem 2.2: Find $C(s)$ such that the steady-state requirements on $e(t)$ are satisfied, and the phase crossover frequency and the gain margin of $L(s)$ are $\omega_{p}$ and GM, respectively.

In some cases, controllers with a richer dynamic structure can be exploited to satisfy further specifications. This is the case of Lead-Lag networks, and PID controllers.

Problem 2.3: Find a controller $C(s)$ that meets the steady-state requirements, and such that the gain crossover frequency, the phase and gain margin are $\omega_{g}$, PM and GM, respectively.

The first step of the design procedure consists in the computation of the gain $K$ of the controller $C(s)$ by im-

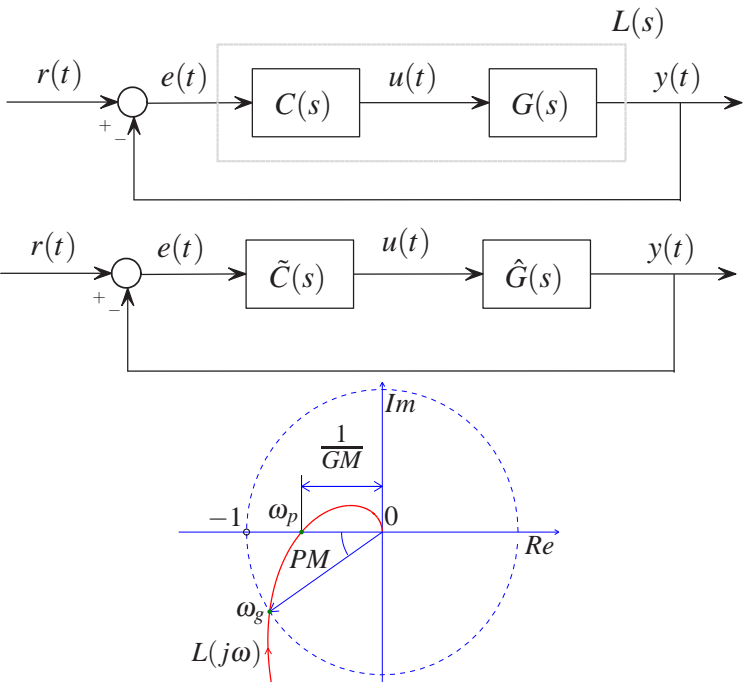

Fig. 1. Classic feedback control architecture and design specifications.

posing the steady-state requirements. This gain can then be considered as part of the plant, i.e., $\hat{G}(s)=K G(s)$, and the dynamical part of the controller $\tilde{C}(s)$ can then be designed. The corresponding equivalent control scheme is the second shown in Fig. 1. To compute the parameters of the compensator to satisfy the specifications of Problem 2.1, the compensator is written as $\tilde{C}(j \omega)=M(\omega) e^{j \varphi(\omega)}$. Imposing $\left|L\left(j \omega_{g}\right)\right|=1$ and $\arg L\left(j \omega_{g}\right)=\mathrm{PM}-\pi$, it is found that

$$
M_{g}=1 /\left|\hat{G}\left(j \omega_{g}\right)\right| \quad \text { and } \quad \varphi_{g}=\mathrm{PM}-\pi-\arg \hat{G}\left(j \omega_{g}\right)
$$

where $M_{g} \stackrel{\text { def }}{=} M\left(\omega_{g}\right)$ and $\varphi_{g} \stackrel{\text { def }}{=} \varphi\left(\omega_{g}\right)$. At this point, since $M_{g}$ and $\varphi_{g}$ are known, by solving the equation

$$
\tilde{C}\left(j \omega_{g}\right)=M_{g} e^{j \varphi_{g}}
$$

via the Inversion Formulae the remaining parameters of $C(s)$ are determined. This approach is anticipated to have a significant graphical interpretation, that provides insight into, and intuitive understanding of, the synthesis method presented here. Indeed, by defining the point $A=\hat{G}\left(j \omega_{g}\right)=$ $M_{A} e^{j \varphi_{A}}$ representing the value of the frequency response of the plant at the desired crossover frequency, and point $B=L\left(j \omega_{g}\right)=e^{j(\pi+\mathrm{PM})}=e^{j \varphi_{B}}$ as the point of the unit circle that the open loop frequency response $L(j \omega)$ has to cross at $\omega=\omega_{g}$ for the specifications in Problem 2.1 to be satisfied, the design task is essentially to determine $\tilde{C}(s)$ such that the equation $A \cdot \tilde{C}\left(j \omega_{g}\right)=B$ holds. Loosely speaking, the desired controller must "bring point $A$ into point $B$ at $\omega=\omega_{g}{ }^{\prime \prime}$. Notice that from (1) there hold $M_{g}=1 / M_{A}$ and $\varphi_{g}=\varphi_{B}-\varphi_{A}$. In the case of Problem 2.2, imposing $\left|L\left(j \omega_{g}\right)\right|=1$ and $\arg L\left(j \omega_{g}\right)=\mathrm{PM}-\pi$, it is found that

$$
M_{p}=1 /\left(\mathrm{GM}\left|\hat{G}\left(j \omega_{p}\right)\right|\right) \quad \text { and } \quad \varphi_{p}=-\pi-\arg \hat{G}\left(j \omega_{p}\right) \text {, }
$$

where $M_{p}=M\left(\omega_{p}\right)$ and $\varphi_{p}=\varphi\left(\omega_{p}\right)$. The equation to be solved has the same structure of (2), with $M_{p}, \varphi_{p}$ and $\omega_{p}$ instead of $M_{g}, \varphi_{g}$ and $\omega_{g}$, respectively. In this case 
$A=\hat{G}\left(j \omega_{p}\right)=M_{A} e^{j \varphi_{A}}$ is the point that has to be brought to point $B=L\left(j \omega_{p}\right)=\mathrm{GM}^{-1}=M_{B} e^{j \pi}$ (which is a point on the real negative half-axis of the Nyquist plane) by the controller, i.e., $A \cdot \tilde{C}\left(j \omega_{p}\right)=B$. This time, $M_{p}=M_{B} / M_{A}$ and $\varphi_{p}=\pi-\varphi_{A}$.

These standard compensators considered here are those found, without exception, in all control feedback design textbooks.

1) Phase-correction networks (with unity DC gain)

- Lead network $C_{\text {Lead }}(s)=\frac{1+\tau s}{1+\alpha \tau s}$;

- Lag network $C_{\mathrm{Lag}}(s)=\frac{1+\alpha \tau s}{1+\tau s}$;

- Lead-Lag network: $C_{\mathrm{LL}}(s)=\frac{s^{2}+2 \zeta_{1} \omega_{n} s+\omega_{n}^{2}}{s^{2}+2 \zeta_{2} \omega_{n} s+\omega_{n}^{2}}$

where $\alpha \in(0,1), \tau>0, \zeta_{1}, \zeta_{2}>0$ and $\omega_{n}>0$. The transfer function of the Lead-Lag network $C_{\mathrm{LL}}(s)$ introduced here generalises the one with real poles and zeros, e.g., [7].

2) PID controllers

- PID controller: $C_{\mathrm{PID}}(s)=K_{p}\left(1+\frac{1}{T_{i} s}+T_{d} s\right)$ with $K_{p}, T_{i}, T_{d}>0$. For details on PI and PD controllers and the corresponding versions with non-negative relative degree, see [10].

\section{PHASE-CORRECTION NETWORKS}

Consider the case of Lead and Lag networks. The design task in this section is to solve Problems 2.1 and 2.2 using a phase-correction network (from now considered to have unity DC gain). In order to solve Problems 2.1 and Problem 2.2 for all types of phase-correction networks, the following result is employed:

Lemma 3.1: ([2], [3]) Solving the equation $\frac{1+j P}{1+j Q}=M e^{j \varphi}$ with $P, Q \in \mathbb{R}, M \in \mathbb{R}_{+}$and $\varphi \in\left(-\frac{\pi}{2}, \frac{\pi}{2}\right)$ yields

$$
P=\frac{M-\cos \varphi}{\sin \varphi} \quad \text { and } \quad Q=\frac{M \cos \varphi-1}{M \sin \varphi}
$$

Lemma 3.1 allows the parameters of the phase-correction network to be computed in closed form.

\section{Lead network: Equations}

$$
\alpha=\frac{M_{g} \cos \varphi_{g}-1}{M_{g}\left(M_{g}-\cos \varphi_{g}\right)} \quad \text { and } \quad \tau=\frac{M_{g}-\cos \varphi_{g}}{\omega_{g} \sin \varphi_{g}}
$$

are called Inversion Formulae for the Lead network. Eq. (2) with $\tilde{C}\left(j \omega_{g}\right)=\bar{C}_{\text {Lead }}\left(j \omega_{g}\right)$ is solvable in $\alpha \in(0,1)$ and $\tau>0$ if and only if the conditions

$$
0<\varphi_{g}<\frac{\pi}{2} \quad \text { and } \quad M_{g}>\frac{1}{\cos \varphi_{g}}
$$

hold. This result is a consequence of Lemma 3.1, with $P=\tau \omega_{g}$ and $Q=\alpha \tau \omega_{g}$. Conditions (5) ensure that $\tau>0$ a) Lead network design

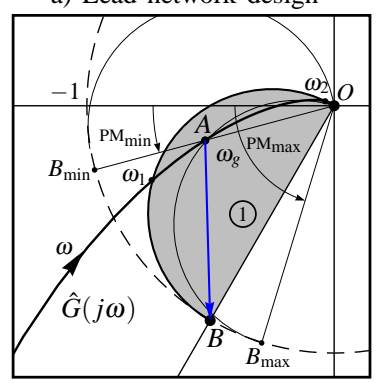

Fig. 2. The two shaded regions in a) and b) on the Nyquist plane represent all points $A$ that can be moved to point $B$ using Lead and Lag networks, see [7].

and $\alpha \in(0,1)$ and can be written as $M_{g}>1$ and $0<$ $\varphi_{g}<\arccos \left(1 / M_{g}\right)$. From these, the minimum and maximum phase margins that are achievable with a Lead network at the frequency $\omega_{g}$ are respectively

$$
\begin{aligned}
& \mathrm{PM}_{\text {min }}=\pi+\arg \hat{G}\left(j \omega_{g}\right) \\
& \mathrm{PM}_{\text {max }}=\pi+\arg \hat{G}\left(j \omega_{g}\right)+\arccos \left(\left|\hat{G}\left(j \omega_{g}\right)\right|\right) .
\end{aligned}
$$

As previously mentioned, the desired gain crossover frequency $\omega_{g}$ defines a point $A$ on the Nyquist plot of the plant, i.e., $A=\hat{G}\left(j \omega_{g}\right)$. The specification on the phase margin defines a point $B$ on the unit circle that the open loop frequency response has to cross at the same frequency, i.e., $B=e^{j(\pi+\mathrm{PM})}=L\left(j \omega_{g}\right)$. The task is to find the compensator such that $\tilde{C}\left(j \omega_{g}\right) \cdot A=B$, so that the network brings point $A$ into point $B$ at the frequency $\omega_{g}$ as required, see Fig. 2a. It can be easily proved that the shaded half-circle shown in Fig. 2a denotes the set of all points $A$ that can be brought to $B$ using a Lead network. Problem 2.1 has a solution if and only if point $A$ belongs to the grey area, i.e., if and only if $\omega_{1} \leq \omega_{g} \leq \omega_{2}$. Fig. 2a also shows how to obtain the points $B_{\max }=e^{j\left(\pi+\mathrm{PM}_{\max }\right)}$ and $B_{\min }=e^{j\left(\pi+\mathrm{PM}_{\min }\right)}$ that correspond to the minimum and maximum phase margins provided by equations (6) and (7).

In the case of Problem 2.2, the solution of (2) with $\tilde{C}\left(j \omega_{p}\right)=\bar{C}_{\text {Lead }}\left(j \omega_{p}\right)$ is given by the same equations written above with $M_{p}, \varphi_{p}$ and $\omega_{p}$ instead of $M_{g}, \varphi_{g}$ and $\omega_{g}$.

Lag network: Equations

$$
\alpha=\frac{M_{g}\left(\cos \varphi_{g}-M_{g}\right)}{1-M_{g} \cos \varphi_{g}} \quad \text { and } \quad \tau=\frac{M_{g} \cos \varphi_{g}-1}{\omega_{g} M_{g} \sin \varphi_{g}} .
$$

are called Inversion Formulae for the Lag network. Eq. (2) with $\tilde{C}\left(j \omega_{g}\right)=\bar{C}_{\mathrm{Lag}}\left(j \omega_{g}\right)$ is solvable if and only if $-\frac{\pi}{2}<\varphi_{g}<0$ and $M_{g}<\cos \varphi_{g}$. This result follows from Lemma 3.1, with $P=\alpha \tau \omega_{g}$ and $Q=\tau \omega_{g}$. These conditions can be also written as $M_{g}<1$ and $-\arccos M_{g}<\varphi_{g}<0$. From these, the minimum and maximum phase margins that are achievable with a Lag network at the frequency $\omega_{g}$ are respectively $\mathrm{PM}_{\min }=\pi+\arg \hat{G}\left(j \omega_{g}\right)-\arccos \left(1 /\left|\hat{G}\left(j \omega_{g}\right)\right|\right)$ and $\mathrm{PM}_{\max }=\pi+\arg \hat{G}\left(j \omega_{g}\right)$. A graphical representations of all the points $A$ that can be brought to point $B$ using a Lag 


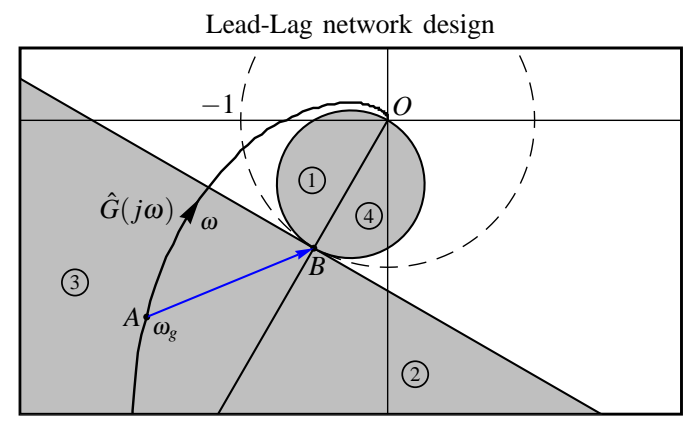

Fig. 3. The shaded region on the Nyquist plane, composed of the two semicircles (1) and (4) and the two half-planes (3) and (2), represents the set of all the points $A$ that can be moved to point $B$ using a Lead-Lag network, see [7].

network is given by the shaded region in Fig. $2 b$. In this case: $i$ ) the admissible region is a quarter of a plane with the origin centred at point $B$; ii) Problem 2.1 has solutions if and only if point $A=\hat{G}\left(j \omega_{g}\right)$ belongs to the shaded area, i.e., if and only if $\omega_{1}<\omega_{g}<\omega_{2}$; iii) the minimum and maximum phase margins $\mathrm{PM}_{\min }$ and $\mathrm{PM}_{\max }$ can be obtained using the graphical construction in Fig. $2 \mathrm{~b}$.

Lead-Lag network: in this case an additional parameter can be exploited to satisfy a further requirement beyond obtaining the desired gain crossover frequency and the phase margin. For example, the value can be assigned of a damping ratio or the natural frequency and solved for the other two parameters. The shaded region on the Nyquist plane shown in Fig. 3 is set of all the points $A$ that can be brought to point $B$ using a Lead-Lag network. An interesting problem for the synthesis of a Lead-Lag network is Problem 2.3, which has been solved graphically and analytically in [7]: let

$$
P_{i}=\frac{2 \zeta_{1} \omega_{i} \omega_{n}}{\omega_{n}^{2}-\omega_{i}^{2}} \quad Q_{i}=\frac{2 \zeta_{2} \omega_{i} \omega_{n}}{\omega_{n}^{2}-\omega_{i}^{2}}, \quad i \in\{g, p\}
$$

Let also $\Phi_{1}=\omega_{g} P_{p}^{-1}-\omega_{p} P_{g}^{-1}, \Phi_{2}=\omega_{p} P_{p}^{-1}-\omega_{g} P_{g}^{-1}$, $\Psi_{1}=\omega_{g} Q_{p}^{-1}-\omega_{p} Q_{g}^{-1}$ and $\Psi_{2}=\omega_{p} Q_{p}^{-1}-\omega_{g} Q_{g}^{-1}$. Problem 2.3 has solutions with a Lead-Lag network if and only if a solution $\omega_{p}$ of

$$
\frac{M_{g}-\cos \varphi_{g}}{\cos \varphi_{g}-M_{g}{ }^{-1}}=\frac{M_{p}-\cos \varphi_{p}}{\cos \varphi_{p}-M_{p}^{-1}}
$$

which is polynomial in $\omega_{p}$, exists such that $\Phi_{1}, \Phi_{2}, \Psi_{1}$ are all positive if $\omega_{p}<\omega_{g}$ and are all negative if $\omega_{p}>\omega_{g}$. In this case the inversion formulae are

$$
\zeta_{1}=\frac{\omega_{g}^{2}-\omega_{p}^{2}}{2 \Phi_{2}} \sqrt{\frac{\Phi_{2}}{\omega_{g} \omega_{p} \Phi_{1}}}, \quad \zeta_{2}=\frac{\omega_{g}^{2}-\omega_{p}^{2}}{2 \Psi_{2}} \sqrt{\frac{\Psi_{2}}{\omega_{g} \omega_{p} \Psi_{1}}}
$$

and $\omega_{n}=\sqrt{\omega_{g} \omega_{p} \Phi_{1} / \Phi_{2}}=\sqrt{\omega_{g} \omega_{p} \Psi_{1} / \Psi_{2}}$. Problem 2.3 admits solutions with a Lead-Lag network with real poles/zeros if and only if a solution $\omega_{p}$ of (10) exists such that $\max \left\{\Phi_{1} \cdot \Phi_{2}, \Psi_{1} \cdot \Psi_{2}\right\}<\omega_{g} \omega_{p}\left(\omega_{g}^{2}-\omega_{p}^{2}\right) / 4$.

\section{DESIGN EXAMPLES USING PHASE-CORRECTION NETWORKS}

This section shows that the method outlined above can be employed to solve many problems that are difficult to address with the standard design techniques, and that can fruitfully be used as written exercises in a basic control course. When the design goal is to solve Problem 2.1 using phasecorrection networks, simple considerations of the frequency response of these compensators suggest that the choice of the type of compensator to use can be made according to Table I.

\begin{tabular}{|c||c|c|}
\hline & $M_{g}>1$ & $M_{g}<1$ \\
\hline \hline$\varphi_{g} \in\left(-\frac{\pi}{2}, 0\right)$ & (4) $\leftrightarrow M_{g} \cos \varphi_{g}>1$ & (2) $\leftrightarrow \cos \varphi_{g}>M_{g}$ \\
Lead-Lag $\left(\zeta_{1}>\zeta_{2}\right)$ & Lag or Lead-Lag $\left(\zeta_{2}>\zeta_{1}\right)$ \\
\hline$\varphi_{g} \in\left(0, \frac{\pi}{2}\right)$ & (1) $\leftrightarrow M_{g} \cos \varphi_{g}>1$ & (3) $\leftrightarrow \cos \varphi_{g}>M_{g}$ \\
Lead or Lead-Lag $\left(\zeta_{1}>\zeta_{2}\right)$ & Lead-Lag $\left(\zeta_{2}>\zeta_{1}\right)$ \\
\hline
\end{tabular}

TABLE I

PHASE CORRECTIONS IN TERMS OF $M_{g}$ AND $\varphi_{g}$ WITH REFERENCES TO

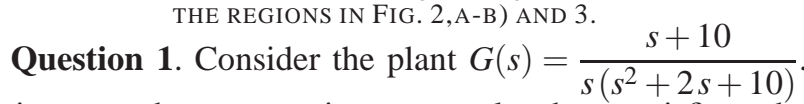
Design a phase-correction network that satisfies the following specifications: velocity constant equal to 1.55 ; phase margin $\mathrm{PM}=45^{\circ}$; gain crossover frequency $\omega_{g}=3.3$ $\mathrm{rad} / \mathrm{sec}$. Find the range of phase margins achievable at $\omega_{g}$.

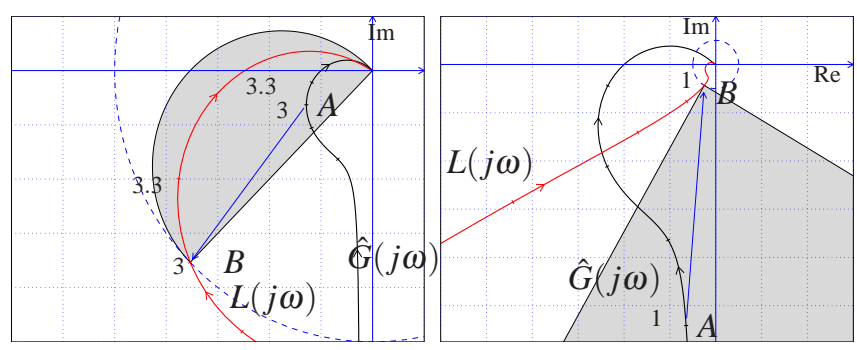

Fig. 4. Graphical representation of solutions of Questions 1-2, respectively.

The DC gain of the network $K$ must be selected so as to satisfy the specification on the velocity constant, i.e., $K_{v}=\lim _{s \rightarrow 0} s C(s) G(s)=K=1.55$. The gain $K$ is now considered to be part of the plant, i.e., $\hat{G}(s)=K G(s)$. To select the right compensation structure, the values $M_{g} \simeq 1.35>1$ and $\varphi_{g} \simeq 34.42^{\circ}$ are computed. These values define the point $A=1 / M_{g} e^{j\left(\pi+\mathrm{PM}-\varphi_{g}\right)} \simeq 0.74 e^{j 190.58^{\circ}}$ that has to be brought to point $B=e^{j(\pi+\mathrm{PM})}=e^{j 225^{\circ}}$ by multiplication with $\tilde{C}\left(j \omega_{g}\right)$. Using Table I or the graphical construction shown at the left of Fig. 4, it is seen that a Lead network can be used. Since (5) are both satisfied, the problem is guaranteed to be solvable. A simple computation gives $\alpha \simeq 0.16$ and $\tau \simeq 0.28 \mathrm{sec}$. The corresponding MATLAB ${ }^{\circledR}$ instructions are shown in Algorithm 1. A graphical plot on the Nyquist plane of $\hat{G}(j \omega)$ and $L(j \omega)$ is shown in Fig. 4. The compensator $C_{\text {Lead }}(s)$ is such that $L(j \omega)$ crosses the point $B=e^{j(P M+\pi)}$ 
at frequency $\omega_{g}$. Using (6) and (7), it is found that the smallest phase margin achievable with a Lead network at $\omega_{g}=3.3 \mathrm{rad} / \mathrm{sec}$ is $\mathrm{PM}_{\min } \simeq 10.58^{\circ}$, and the largest phase margin is $\mathrm{PM}_{\max } \simeq 52.62^{\circ}$.

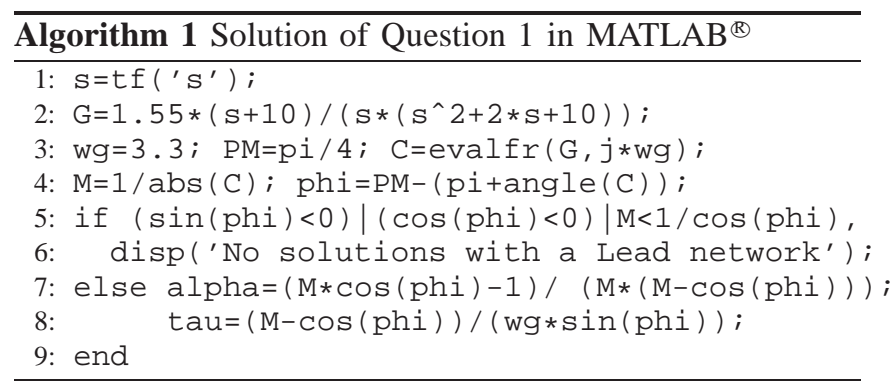

Question 2. Design a phase-correction network that meets the following specifications: velocity error equal to $0.1 ; \mathrm{PM}=60^{\circ} ; \omega_{g}=1 \mathrm{rad} / \mathrm{sec}$. Find the range of phase margins achievable at this crossover frequency with this network.

Since the velocity error is $e_{v}=1 / K_{v}$ and $K_{v}=K$ as shown in Question 1, it is found $K=10$. Let also $\hat{G}(s)=K G(s)$. To select the right compensator, the values $M_{g}=\sqrt{85 / 101} / 10 \simeq 0.092<1$ and $\varphi_{g}=-\pi / 6-\arctan (1 / 10)+\arctan (2 / 9) \simeq-23.18^{\circ}$ are calculated, which lead to $A=\hat{G}\left(j \omega_{g}\right)=M_{A} e^{j \varphi_{A}}=10.9 e^{j 163.18^{\circ}}$ and $B=e^{j(\pi+P M)}=M_{B} e^{j \varphi_{B}}=e^{j 240^{\circ}}$. Thus, from Table I or the graphical construction shown to the right of Fig. 4, it is seen that a Lag network should be used. Since $-\arccos \left(M_{g}\right)<\varphi_{g}<0$, a Lag network solving the problem exists with $\alpha=0.083$ and $\tau=25.36$ sec by substituting the values of $M_{g}$ and $\varphi_{g}$ thus found in (8). The smallest phase margin achievable with a Lag network at $\omega_{g}=1$ $\mathrm{rad} / \mathrm{sec}$ is $\mathrm{PM}_{\min } \simeq-1.55^{\circ}$, and the largest phase margin is $\mathrm{PM}_{\max } \simeq 83.18^{\circ}$.

Question 3. Design a Lead-Lag network that meets the following specifications: velocity constant equal to 0.1 ; $\mathrm{PM}=45^{\circ} ; \mathrm{GM}=3 ; \omega_{g}=1 \mathrm{rad} / \mathrm{sec}$.

The DC gain of the Lead-Lag network $K$ must satisfy the specification on the velocity constant $K_{v}=$ $\lim _{s \rightarrow 0} s C_{\mathrm{LL}}(s) G(s)=\lim _{s \rightarrow 0} s K G(s)=K=0.1$. Now, consider this gain to be part of the plant, and define $\hat{G}(s)=$ $K G(s)$. We get $M_{g}=(1 / K) \sqrt{85 / 101} \simeq 9.17$ and $\varphi_{g}=$ $-\pi / 4-\arctan (1 / 10)+\arctan (2 / 9) \simeq 38.18^{\circ}$. Hence

$$
\gamma \stackrel{\text { def }}{=} C \frac{M_{g}-\cos \varphi_{g}}{\cos \varphi_{g}-M_{g}^{-1}}=\frac{170-103 \sqrt{2} K}{103 \sqrt{2} K-202 K^{2}} \simeq 12.38 \text {. }
$$

Now, $M_{p}$ and $\varphi_{p}$ are expressed as a function of $\omega_{p}$, i.e., $M_{p}=$ $\omega_{p} \Gamma_{p} /\left(G M \cdot K \Pi_{p}\right)$ and $\cos \varphi_{p}=\omega_{p}\left(\omega_{p}^{2}+10\right) /\left(\Gamma_{p} \Pi_{p}\right)$, where $\Gamma_{p}=\sqrt{4 \omega_{p}^{2}+\left(10-\omega_{p}^{2}\right)^{2}}$ and $\Pi_{p}=\sqrt{\omega_{p}^{2}+100}$. Plugging these into (10) yields

$$
\omega^{6}-(16+H+\gamma H) \omega^{4}+10(10-H-\gamma H) \omega^{2}+100 \gamma H^{2}=0,
$$

where $H=\mathrm{GM} \cdot K=0.3$. This biquadratic equation has two positive solutions: $\omega_{p}^{\prime}=3.96$ and $\omega_{p}^{\prime \prime}=2.37$. Using the first solution, positive values for $\Phi_{1}, \Phi_{2}, \Psi_{1}$ and $\Psi_{2}$ are obtained. Therefore, the condition of existence of a suitable Lead-Lag network are not satisfied. If $\omega_{p}=\omega_{p}^{\prime \prime}=2.37$ is used, those values are all negative, and therefore they satisfy the condition of existence of a Lead-Lag network. The corresponding values of the parameters of the network are $\zeta_{1}=20.75, \zeta_{2}=1.67$ and $\omega_{n}=0.30 \mathrm{rad} / \mathrm{sec}$. Since $\zeta_{1}$ and $\zeta_{2}$ are both greater than 1 , the solution can be also given in terms of a Lead-Lag network with real poles/zeros.

\section{PID CONTROLLERS}

Because of the pole at the origin in the transfer function of the standard PID controller, it is essential to discriminate between the case where the steady-state specifications impose a constraint on the integration constant $K_{i}$ and the case where the presence of the pole at the origin in the PID controller alone is sufficient to satisfy the steady-state requirements. In the first case, it is shown in [10] that three simple formulae yield the expression of the three parameters of the PID controller as a function of the phase margin and the crossover frequency required. In the solution of the second problem there is a degree of freedom that can be exploited to satisfy additional requirements. In [10], two possibilities were considered. The first is the imposition of the ratio of the two time constants of the PID controllers, which is useful since that ratio directly affects the quality of the time response of the closed-loop system, and its assignment can avoid the situation of complex conjugate zeros in the transfer function of the PID controller. The second is the imposition of the gain margin, in addition to the phase margin. Indeed, in the case of specifications on both stability margins and on the gain crossover frequency, it is possible to compute the phase crossover frequency by solving a polynomial equation, and then compute the parameters of the controller in finite terms.

\section{DESIGN EXAMPLES USING PID CONTROLLERS}

Consider the feedback control scheme in Question 1.

Question 4. Design a compensator that meets the following specifications: zero velocity error; phase margin equal $\mathrm{PM}=45^{\circ}$; gain crossover frequency $\omega_{g}=3 \mathrm{rad} / \mathrm{sec}$.

The steady-state specification is automatically satisfied by using a PID controller. The extra freedom can be used to select $T_{i} / T_{d}$. Choose e.g. $T_{i} / T_{d}=8$. Then, the values $M_{g}=|G(3 j)|^{-1}=3 \sqrt{37 / 109} \simeq 1.75$ and $\varphi_{g}=7 \pi / 4-\arctan (3 / 10)+\arctan 6 \simeq 18.84^{\circ}$ are computed. Since $\varphi_{g} \in(-\pi / 2, \pi / 2)$, the problem is guaranteed to admit a solution, see Theorem 1 in [10]. Using (12-14) in [10] it is found $K_{p}=1.65, T_{i}=1.50 \mathrm{sec}$ and $T_{d}=0.19 \mathrm{sec}$. A graphical representation of the design procedure is shown in the first of Fig. 5. The controller brings point $A=G\left(j \omega_{g}\right)$ 

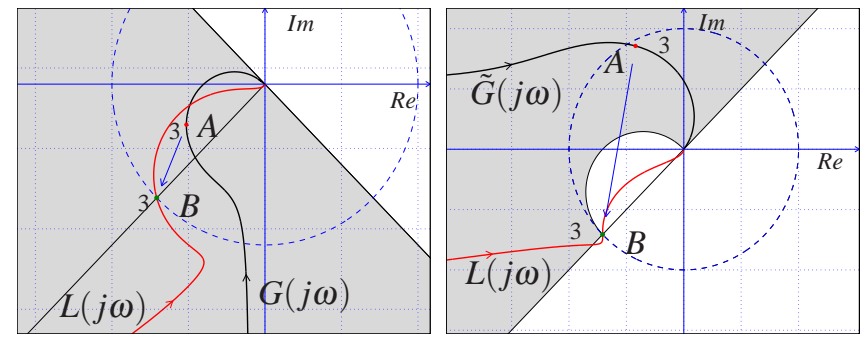

Fig. 5. Design of PID controller on the Nyquist plane for Questions 4,5.

to $B=e^{j(\mathrm{PM}+\pi)}$. The shaded area represents the set of all the admissible points $A$ that can be brought to $B$ by using a PID controller.

Question 5. Design a compensator that meets the following specifications: zero velocity error, acceleration error not greater than $0.2 ; \mathrm{PM}=45^{\circ} ; \omega_{g}=3 \mathrm{rad} / \mathrm{sec}$.

As already observed, the steady-state requirement in this case imposes $K_{i}=K_{p} / T_{i} \geq 5$. Let us choose $K_{i}=$ 5. Hence, $M_{g}=\omega_{g} /\left(K_{i}\left|G\left(j \omega_{g}\right)\right|\right) \simeq 1.05$ and $\varphi_{g}=\mathrm{PM}-$ $\pi / 2-\arg G\left(j \omega_{g}\right) \simeq 108.84^{\circ}$. The conditions $0<\varphi_{g}<\pi$ and $M_{g} \cos \varphi_{g}<1$ are both satisfied, so that the problem admits solutions, see Theorem 3 in [10]. Using (35-37) in [10], it is found that $T_{i} \simeq 0.33 \mathrm{sec}, T_{d} \simeq 0.45 \mathrm{sec}$, and $K_{p}=K_{i} T_{i} \simeq 1.65$. Since in this case $T_{i}<4 T_{d}$, the zeros of the compensator are complex conjugate and equal to $-1.11 \pm 2.34 j$. The Nyquist plot of $\tilde{G}(j \omega)=\frac{K_{i}}{s} G(s)$ is shown in the second of Fig. 5. The shaded area of the figure describes the set of all the points $A$ that can be brought to $B$ by using the transfer function $s C_{\mathrm{PID}}(s) / K_{i}=1+T_{i} s+T_{1} T_{d} s^{2}$.

\section{PRACTICAL IMPLICATIONS}

The Inversion Formulae method for the synthesis of standard controllers is currently used by a number of lecturers in control courses in Computer Science, Mechanics, Electronic and Telecommunication Engineering degree programs in a number of Italian universities. An example of this is the course Automatic Control at the University of Modena, taught to for students enrolled in the second year of Electrical Engineering (around 50 students every year), and to those enrolled in the third year of Computer Engineering (around 80 every year). Since 2011, this course has been organised in 90 hours of lectures/tutorials and is worth nine $C F U$ (crediti formativi universitari). These credits are transferable through the European Credit Transfer System (ECTS). This is a standard fundamental course where the classic foundations of classic control theory are addressed. The course has three main parts. The first focuses on the analysis of linear dynamical single-input single-output systems and the second deals with the synthesis of compensators. The final part involves the teaching of non-linear methods as well as discrete systems. Student reviews for this course are overwhelmingly positive. In the Academic Year 2011/2012, the student score for the course Automatic Control was 8.67 out of 10 , which is significantly higher than the average score for all courses (7.82). In the Academic Year 2010/2011, the student score was 8.97 out of 10 , which was not only higher than the average score for all courses (7.81), but was also the highest score for all courses. This indicates a qualitative score of 'above average' for the last two year in which it has been taught in the current format.

The Inversion Formulae method is also currently being taught in the Automatic Systems course in the Electronic and Telecommunication track at the 'E. Fermi' High School in Modena, Italy. This is a five-year Industrial Technical Institute (years 9 to 14 in the Italian secondary education plan). The lecture topics on the Inversion Formulae method were:: Bode and Nyquist diagrams and stability margins; description of the analytical and graphical solutions through numerical examples; and execution of the proposed algorithms using MATLAB $^{\circledR}$.

\begin{tabular}{|c|c|c|c|}
\hline$Q_{1}$ & $\begin{array}{c}\text { Item } \\
\text { Did the design procedure help you improve } \\
\text { your general knowledge of compensator design? }\end{array}$ & $100 \%$ & $0 \%$ \\
\hline$Q_{2}$ & $\begin{array}{l}\text { Do you think that the graphical interpretation in } \\
\text { the Nyquist plot helps you to better understand } \\
\text { the role of the controller in a feedback scheme? }\end{array}$ & $92.9 \%$ & $7.1 \%$ \\
\hline$Q_{3}$ & $\begin{array}{c}\text { Are you able to select the controller which solves } \\
\text { the design problems by drawing the allowable } \\
\text { domains with compass and ruler? }\end{array}$ & $84.6 \%$ & $15.4 \%$ \\
\hline$Q_{4}$ & $\begin{array}{c}\text { Do you think that writing the design algorithms } \\
\text { in a lab activity makes your knowledge long lasting? }\end{array}$ & $85.7 \%$ & $14.3 \%$ \\
\hline$Q_{5}$ & $\begin{array}{c}\text { Do you think that this method } \\
\text { is simple enough to be used in high school courses? }\end{array}$ & $86.7 \%$ & $13.3 \%$ \\
\hline$Q_{6}$ & $\begin{array}{l}\text { Do you think that with this method PID controllers } \\
\text { could be introduced in a high school programme? }\end{array}$ & $91.7 \%$ & $8.3 \%$ \\
\hline \multicolumn{4}{|c|}{ TABLE II } \\
RESPONSES TO THE QUESTIONNAIRE
\end{tabular}

After this set of lectures, students were asked to answer an anonymous questionnaire; their responses are shown in Table II. The results are highly satisfactory. In particular they indicate that during the process of learning of the method, students perceive that their general knowledge of control feedback design improves, see $Q_{1}-Q_{2}$, and that the method provides new skills, see $Q_{3}$. Students are aware that laboratory activities and writing algorithms to solve the problems gave them a long-lasting understanding of the underlying principles, see $Q_{2}-Q_{4}$. This result confirms the theories of constructivism related to computer scaffolding, CSCL (computer-supported collaborative learning) and CSILE (computer-supported intentional learning environments), [11], [12]. Finally, students agree that the method is simple and can be used in basic control courses, see $Q_{2}$ $Q_{4}$.

To evaluate and compare the students' confidence and level of understanding of the Inversion Formulae design technique against the more traditional technique based on trial-and-error procedures, another test was undertaken on a different class of students enrolled in the same course. These students were divided into two groups with equivalent 

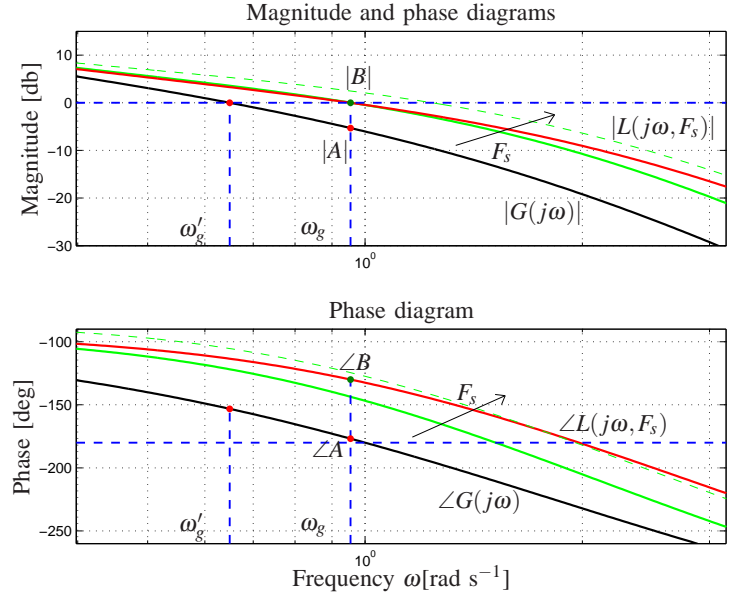

Fig. 6. Test Question. The Bode plots of $G(j \omega)$ (continuous thick line) and the closed-loop frequency responses using classical Bode solution (thin lines) and Inversion Formulae method (dashed line)

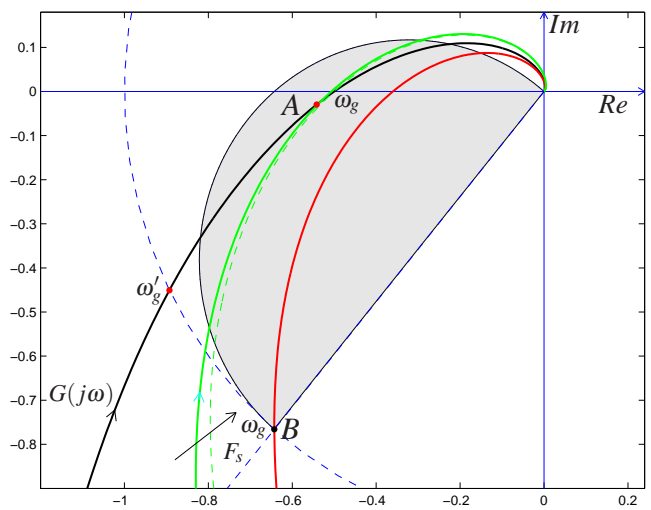

Fig. 7. Test Question. The Nyquist plots of $G(j \omega)$ (continuous thick line) and the closed-loop frequency responses using classical Bode solution (thin lines) and Inversion Formulae method (dashed line).

prior knowledge of the subject. The first group was asked to solve a control feedback design problem using the classical method on the Bode plot, and the second was required to employ the Inversion Formulae method to solve the same problems. They were asked to address the same set of control problems in a written test. Moreover, the second (Inversion Formulae) group was given considerably less time to solve the problems. A sample test problem follows:

Test Question. Consider the plant described by the transfer function $G(s)=\frac{5}{s(s+1)(s+2)(s+3)}$, which already includes the gain and the required integrators to satisfy the steadystate specification. Design a Lead network that yields a phase margin of $50^{\circ}$.

Classical solution on Bode diagram. The design of $C_{\text {Lead }}(s)$ is obtained by selecting the pole and zero of the compensator in such a way that the maximum phase lead $\Phi_{\max }$ of the compensator is placed at the gain crossover frequency of the uncompensated system. The undesired

\begin{tabular}{|ccc|}
\hline & Classical solution & Inversion Formulae \\
\hline \hline Mean & 6.04 & 7.38 \\
\hline Standard Error & 0.57 & 0.35 \\
\hline Median & 6.5 & 7.5 \\
\hline Minimum & 3 & 5 \\
\hline Maximum & 8.5 & 9 \\
\hline Count & 13 & 13 \\
\hline Standard Deviation & 1.39 & 1.26 \\
\hline Sample Variance & 1.94 & 1.59 \\
\hline
\end{tabular}

TABLE III

DESCRIPTIVE STATISTICAL VALUE

shift in the magnitude of the loop gain, which causes the phase margin of the controlled system to be different from this value $\Phi_{\max }$, is compensated using a trial-anderror procedure for the selection of a safety factor $F_{S}$ by rule of thumb. Students can estimate the uncompensated phase margin $\phi_{G}=26.8^{\circ}$ and the gain crossover frequency $\omega_{g^{\prime}}=0.65 \mathrm{rad} / \mathrm{sec}$ by drawing the Bode diagrams of $G(j \omega)$, see Fig. 6. The parameter $\alpha$ of $C_{\text {Lead }}(s)$ is then calculated using the well-known formula $\alpha=\frac{1-\sin \Phi_{\max }}{1+\sin \Phi_{\max }}$, where $\Phi_{\max }$ is the maximum network phase shift. This is designed in such a way that at $\omega=\omega_{g^{\prime}}$ the phase margin is PM, i.e. $\Phi_{\max }=\mathrm{PM}-\phi_{G}+F_{s}$. Since at frequency $\omega_{g^{\prime}}$ the gain of the controller is not equal to $0 \mathrm{db}$, as a rule of thumb a safety factor $F_{s}$ of about $10^{\circ}$ is introduced. For $F_{s}=10^{\circ}$, gives the value $\alpha=0.29$. The value $\tau=\frac{1}{\omega_{g} \sqrt{\alpha}}=1.94 \mathrm{sec}$ is then designed in such a way that the compensated system has a magnitude of $0 \mathrm{db}$ at $\omega_{g}=0.95 \mathrm{rad} / \mathrm{sec}$, where $\omega_{g}$ satisfies $\left|G\left(j \omega_{g}\right)\right|=10 \log _{10} \alpha$. The Bode and the Nyquist diagrams of the corresponding loop gain frequency responses are shown with the solid thin lines in Figs. 6 and 7. Notice that the phase margin of the compensated system is only $36.1^{\circ}$. This value can be increased choosing a higher value of $F_{s}$ by several iterations of the method, but the compensated phase margin for $F_{s} \in\left[15^{\circ}: 5^{\circ}: 30^{\circ}\right]$ is still very low, see the dotted thin lines in Figs. 6-7.

Solution using Inversion Formulae. The design phase margin PM defines the point $B=e^{j 230^{\circ}}$. Given a further design specification on the gain crossover frequency $\omega_{g}=$ $0.95 \mathrm{rad} / \mathrm{sec}$, the magnitude and the phase of $B / G\left(j \omega_{g}\right)$ are $M_{g}=0.54$ and $\varphi_{g}=183^{\circ}$, respectively. Using Table 1 we see that a Lead network can indeed solve the problem. Substituting $M_{g}$ and $\varphi_{g}$ in (4), gives the values $\alpha=0.12$, $\tau=1.66 \mathrm{sec}$. The phase margin of the controlled system is exactly $50^{\circ}$, see dashed lines in Fig. 6 and Fig. 7.

The test results obtained using a graded scale $1-10$ are shown in Table III, where a statistical analysis is carried out. This result opens new interesting scenarios for the introduction of the proposed method in undergraduate control textbooks.

\section{CONCLUSION}

This paper has presented a design method for all types of standard compensators taught in control courses, which represent the vast majority of compensators used in industry. 
This technique - employed in several universities in Italy in a number of years - does not rely on iterative procedures on Bode or Nyquist plots, and appears to be very suitable for numerical exercises that can test students' skills in every aspect of the compensator design process. However, the value of this method lies in its flexibility to adapt to different design scenarios, including PI and PD controllers, PID controllers with an additional pole introduced in the derivative action, discrete time counterparts, and systems with delays, [10], [13]. The relevance of the Inversion Formulae in the context of written exercises has been demonstrated with a number of examples of questions that are extremely difficult to tackle with the standard approaches.

\section{ACKNOWLEDGEMENT}

The authors are grateful to Dr. Daniel Southam, Prof. Arnaldo Losi and Prof. Maria Cristina Zanti for their advice on the topic of this paper.

\section{REFERENCES}

[1] K.J. Åström, and T. Hagglund, Advanced PID Control. Instrument Society of America, Research Triangle Park, NC, 2006.

[2] C.L. Phillips. Analytical Bode Design of Controllers. IEEE T. Educ., vol. 28, pp. 43-44, 1985.

[3] R. Zanasi and G. Marro, "New formulae and graphics for compensator design." Proc. IEEE Int. Conf. Contr. Applic., vol. 1, pp. 129-133, 1998.

[4] C.L. Phillips, and H.T. Nagle, Digital Control Systems. Analysis and Design. Prentice-Hall, 1995.

[5] G. Marro, Controlli Automatici. Zanichelli Ed., Bologna (Italy), 2004.

[6] A. Ferrante, A. Lepschy, and U. Viaro. Introduzione ai Controlli Automatici. UTET Università, 2000.

[7] R. Zanasi, S. Cuoghi, and L. Ntogramatzidis, "Analytical and Graphical Design of Lead-Lag Compensators". International Journal of Control, vol. 84, pp.1830-1846, 2011.

[8] M. Mernik, V. Žumer, "An educational tool for teaching compiler construction". IEEE T. Educ., vol. 46, pp. 61-68, 2003.

[9] S. Dormido et al. "The role of interactivity in control learning". Int. J. Engng. Ed., vol. 21, pp. 1122-1133, 2005.

[10] L. Ntogramatzidis, and A. Ferrante, "Exact Tuning of PID Controllers in Control Feedback Design", IET Control Theory A., vol. 5, pp. 565-578, 2011.

[11] N.W. Holmes, "The myth of the educational computer". Computer, vol. 32, pp. 36-42, 1999.

[12] L. Harasim, "A framework for on line learning: The virtual-U". Computer, vol. 32, pp. 44-49, 1999.

[13] R. Zanasi and R. Morselli, "Discrete Inversion Formulas for the Design of Lead and Lag Discrete Compensators." Proc. European Contr. Conf. 2009, pp. 5069-5074, Aug. 23-26, 2009.

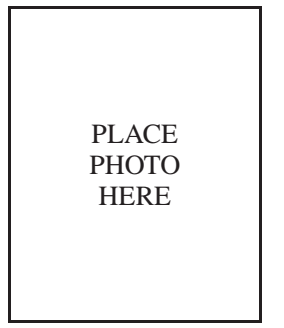

Lorenzo Ntogramatzidis Lorenzo Ntogramatzidis received his "Laurea" degree, cum laude, in computer engineering in 2001 from the University of Bologna, Italy. He received his $\mathrm{Ph} . \mathrm{D}$. in control and operations research in 2005 from the same university. From 2005 to 2008, he was a post-doctoral Research Fellow at the Department of Electrical and Electronic Engineering, The University of Melbourne, Australia. Since 2009, he has been with the Department of Mathematics and Statistics at Curtin University, Perth, Australia, where he is currently Senior Lecturer. His research interests are in the area of systems and control theory.

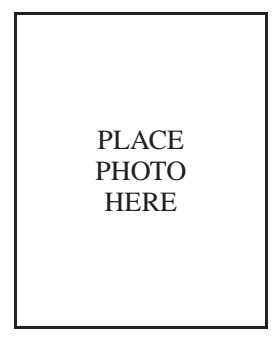

Roberto Zanasi Roberto Zanasi graduated with honors in electrical engineering in 1986. Since 1998 he has been working as Associate Professor of Automatic Control at the University of Modena and Reggio Emilia. In 2004 he became Professor in Automatic Control. He held the position of Visiting Scientist at the IRIMS of Moscow in 1991, at the MIT in Boston in 1992 and at the Universite Catholique de Louvain in 1995. His research interests include mathematical modeling, simulation, automotive, control of variablestructure systems, integral control, sliding mode control and robotics.

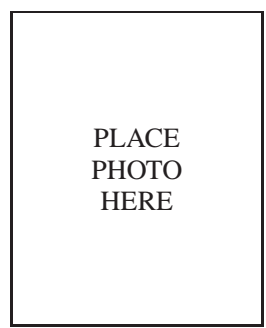

Stefania Cuoghi Stefania Cuoghi received the Laurea degree in electronic engineering in 1999 from the University of Bologna, Italy. She received her Ph.D. in control research in 2012 from the University of Modena and Reggio Emilia, Italy. Her current research interests include mathematical modelling, simulation, analytical and graphical design of regulators for linear control and robotics. 